\title{
INEQUALITIES FOR THE NORM AND NUMERICAL RADIUS FOR HILBERT $C^{*}$-MODULE OPERATORS
}

\begin{abstract}
In this paper, we introduce some inequalities between the operator norm and the numerical radius of adjointable operators on Hilbert $C^{*}$-module spaces. Moreover, we establish some new refinements of numerical radius inequalities for Hilbert space operators. More precisely, we prove that if $T \in B(H)$ and

$$
\min \left(\frac{\left\|T+T^{*}\right\|^{2}}{2}, \frac{\left\|T-T^{*}\right\|^{2}}{2}\right) \leq \max \left(\inf _{\|x\|=1}\|T x\|^{2}, \inf _{\|x\|=1}\left\|T^{*} x\right\|^{2}\right),
$$
\end{abstract}

then

$$
\|T\| \leq \sqrt{2} \omega(T) ;
$$

this is a considerable improvement of the classical inequality

$$
\|T\| \leq 2 \omega(T) .
$$

Key words: Bounded linear operator, Hilbert space, Norm inequality, Numerical radius

2010 Mathematical Subject Classification: Primary 47A12; Secondary 47 A30

1. Introduction and preliminaries. Let $B(H)$ denote the $C^{*}$-algebra of all bounded linear operators on a complex Hilbert space $H$ with the inner product $\langle\cdot, \cdot\rangle$. If $\operatorname{dim} H=n$, we identify $B(H)$ with the space $M_{n}$ of all $n \times n$ matrices with entries in the complex field. For $T \in B(H)$, let $\|T\|$ denote the usual operator norm and

$$
\omega(T)=\sup \{|\langle T x, x\rangle|:\|x\|=1\}
$$

denote the numerical radius of $T$. It is well known that $\omega(\cdot)$ is a norm on $B(H)$ and that

$$
\frac{\|T\|}{2} \leq \omega(T) \leq\|T\|
$$

(C) Petrozavodsk State University, 2020 
for all $T \in B(H)$. The first inequality becomes an equality if $T^{2}=0$ (use the first Kittaneh inequality below). The second inequality becomes an equality if $T$ is normal. Recently, the authors of [13] tried to show that $\|T\| \leq \sqrt{2} \omega(T)$ holds, whenever $T$ is an invertible operator. However, Cain [1] constructed some counterexamples. Several numerical radius inequalities improving those in (1) have been recently given in [2-5], [11], and [14]. For instance, Dragomir proved that

$$
\omega^{2}(T) \leq \frac{1}{2}\left(\omega\left(T^{2}\right)+\|T\|^{2}\right)
$$

for any $T \in B(H)$. And Kittaneh proved that, for any $T \in B(H)$,

$$
\omega(T) \leq \frac{1}{2}\left(\|T\|+\left\|T^{2}\right\|^{\frac{1}{2}}\right)
$$

and

$$
\frac{\left\|T T^{*}+T^{*} T\right\|}{4} \leq \omega^{2}(T) \leq \frac{\left\|T T^{*}+T^{*} T\right\|}{2} .
$$

Theese inequalities can be found in [9], [10], respectively. Furthermore, Holbrook in [7] showed that, for any $R, S \in B(H)$,

$$
\omega(R S) \leq 4 \omega(R) \omega(S)
$$

and

$$
\omega(R S) \leq 2 \omega(R) \omega(S)
$$

when $R S=S R$.

See [6] for other results and historical comments on the numerical radius. Now, here is a reminder of the definition of a Hilbert module, according to [12].

Let $\mathcal{A}$ be a $C^{*}$-algebra (not necessarily unital or commutative). An inner-product $\mathcal{A}$-module is a linear space $E$, which is a right $\mathcal{A}$-module (with a compatible scalar multiplication: $\lambda(x a)=x(\lambda a)=(\lambda x) a$ for all $x \in E, a \in \mathcal{A}$ and $\lambda \in \mathbb{C})$, together with a map $\langle\cdot, \cdot\rangle: E \times E \longrightarrow \mathcal{A}$, such that

(i) $\langle x, x\rangle \geqq 0$, meaning it is one of the positive operators in $\mathcal{A}$; $\langle x, x\rangle=0$ iff $x=0$,

(ii) $\langle x, \lambda y+z\rangle=\lambda\langle x, y\rangle+\langle x, z\rangle$,

(iii) $\langle x, y a\rangle=\langle x, y\rangle a$, 
(iv) $\langle x, y\rangle=\langle y, x\rangle^{*}$,

for all $x, y, z \in E, a \in \mathcal{A}, \lambda \in \mathbb{C}$.

For $x \in E$, we write $\|x\|=\|\langle x, x\rangle\|^{\frac{1}{2}}$. An inner-product $\mathcal{A}$-module that is complete with respect to its norm is called a Hilbert $\mathcal{A}$-module, or a Hilbert $C^{*}$-module, over the $C^{*}$-algebra $\mathcal{A}$. We denote, by $L(E)$, the $C^{*}$-algebra of all adjointable operators on $E$ (i. e., of all maps $T: E \longrightarrow E$, such that there exists a $T^{*}: E \longrightarrow E$ with the property $\langle T(x), y\rangle=$ $=\left\langle x, T^{*}(y)\right\rangle$, for all $\left.x, y \in E\right)$ and let $L^{-1}(E)$ denote the set of all invertible operators in $L(E)$.

Definition 1. For $T \in L(E)$, let

$$
\begin{gathered}
\delta(T)=\sup \{\|\langle T x, x\rangle\|:\|x\|=1\}, \\
\|T\|=\sup \{\|T x\|:\|x\|=1\}
\end{gathered}
$$

respectively, denote the numerical radius and operator norm of $T$.

Recently, in [15], we have shown that

$$
\|T\| \leq 2 \delta(T)
$$

and

$$
\delta(T S) \leq 4 \delta(T) \delta(S)
$$

We are able to improve the inequalities (4) and (5). The results in this paper considerably improve inequalities (1) and (2).

2. Main results. Let $T \in L(E)$. For the sake of convenience, we prepare the following notation:

$$
m(T)=\min \left(\frac{\left\|T-T^{*}\right\|^{2}}{2}, \frac{\left\|T+T^{*}\right\|^{2}}{2}\right)
$$

and

$$
M(T)=\max \left(\inf _{\|x\|=1}\|T x\|^{2}, \inf _{\|x\|=1}\left\|T^{*} x\right\|^{2}\right) .
$$

In order to derive our main results, we need the following lemmas.

Lemma 1. If $T \in L(E)$ is self-adjoint, then

$$
\delta(T)=\|T\| .
$$

Proof. First, we show that the result holds for positive operators. 
Let $G \in L(E)$ be positive. Since $\mathrm{L}(\mathrm{E})$ is a $C^{*}$-algebra, we know that $\left\|G^{*} G\right\|=\|G\|^{2}$. Then,

$$
\left\|G^{*} G\right\|=\sup _{\|x\|=1}\left\|\left\langle G^{*} G x, x\right\rangle\right\| .
$$

Replacing $G$ by $\sqrt{G}$ gives

$$
\|G\|=\sup _{\|x\|=1}\|\langle G x, x\rangle\| .
$$

Now, let $T \in L(E)$ be just self-adjoint. By Proposition 1.1 in [12],

$$
\delta(T)=\sup _{\|x\|=1}\|\langle T x, x\rangle\| \leq\|T\| .
$$

On the other hand, being self-adjoint, $T$ can be decomposed: $T=T_{+}-T_{-}$, such that $T_{+}$and $T_{-}$are both positive and $T_{+} T_{-}=T_{-} T_{+}=0$, and also $\|T\|=\max \left(\left\|T_{+}\right\|,\left\|T_{-}\right\|\right)$. Note that

$$
\left.\sup _{\|x\|=1}\left\|\left\langle T_{+}^{3} x, x\right\rangle\right\|=\left\|T_{+}^{3}\right\|, \quad \text { (by }(7)\right) ;
$$

then there exist a sequence $\left\{x_{n}\right\}$ of unit vectors in $E$, such that

$$
\left\|T_{+}^{3}\right\|=\lim _{n \rightarrow \infty}\left\|\left\langle T_{+}^{3} x_{n}, x_{n}\right\rangle\right\|
$$

Therefore,

$$
\begin{gathered}
\sup _{\|x\|=1}\|\langle T x, x\rangle\| \geq\left\|\left\langle T\left(\frac{T_{+} x_{n}}{\left\|T_{+} x_{n}\right\|}\right), \frac{T_{+} x_{n}}{\left\|T_{+} x_{n}\right\|}\right\rangle\right\|= \\
=\left\|\left\langle\left(T_{+}-T_{-}\right)\left(\frac{T_{+} x_{n}}{\left\|T_{+} x_{n}\right\|}\right), \frac{T_{+} x_{n}}{\left\|T_{+} x_{n}\right\|}\right\rangle\right\|= \\
=\frac{\left\|\left\langle T_{+}^{3} x_{n}, x_{n}\right\rangle\right\|}{\left\|T_{+} x_{n}\right\|^{2}} \geq \frac{\left\|\left\langle T_{+}^{3} x_{n}, x_{n}\right\rangle\right\|}{\|T\|^{2}}
\end{gathered}
$$

and so:

$$
\sup _{\|x\|=1}\|\langle T x, x\rangle\| \geq \frac{\left\|T_{+}\right\|^{3}}{\|T\|^{2}}=\lim _{n \rightarrow \infty} \frac{\left\|\left\langle T_{+}^{3} x_{n}, x_{n}\right\rangle\right\|}{\|T\|^{2}} .
$$

Similarly,

$$
\sup _{\|x\|=1}\|\langle T x, x\rangle\| \geq \frac{\left\|T_{-}\right\|^{3}}{\|T\|^{2}} .
$$


By (9) and (10),

$$
\delta(T)=\sup _{\|x\|=1}\|\langle T x, x\rangle\| \geq \max \left(\frac{\left\|T_{+}\right\|^{3}}{\|T\|^{2}}, \frac{\left\|T_{-}\right\|^{3}}{\|T\|^{2}}\right)=\|T\| .
$$

The result follows from inequalities (8) and (11).

Lemma 2. If $T \in L(E)$, then

(a) $m(T) \leq 2 \delta^{2}(T)$.

(b) $M(T)=\frac{1}{\left\|T^{-1}\right\|^{2}}$, if $T$ is invertible.

Proof. (a) Since $T+T^{*}$ is self-adjoint, from Lemma 1 we have:

$$
\left\|T+T^{*}\right\|=\delta\left(T+T^{*}\right)
$$

So,

$$
\frac{\left\|T+T^{*}\right\|^{2}}{2}=\frac{\left(\delta\left(T+T^{*}\right)\right)^{2}}{2} \leq \frac{\left(\delta(T)+\delta\left(T^{*}\right)\right)^{2}}{2}=2 \delta^{2}(T) .
$$

Consequently,

$$
\frac{\left\|T+T^{*}\right\|^{2}}{2} \leq 2 \delta^{2}(T)
$$

Since $m(T) \leq \frac{\left\|T+T^{*}\right\|^{2}}{2}$, the result follows from (12).

(b) See [8, p. 41].

Lemma 3. Let $E$ be a Hilbert $C^{*}-$ module. Then

$$
\|\langle a, a\rangle+\langle b, b\rangle\| \leq \frac{1}{2}\left(\|a+b\|^{2}+\|a-b\|^{2}\right),
$$

for any $a, b \in E$.

Proof. Suppose that $a, b \in E$; then

$$
\begin{aligned}
& \langle a+b, a+b\rangle=\langle a, a\rangle+\langle a, b\rangle+\langle b, a\rangle+\langle b, b\rangle, \\
& \langle a-b, a-b\rangle=\langle a, a\rangle-\langle a, b\rangle-\langle b, a\rangle+\langle b, b\rangle .
\end{aligned}
$$

Thus,

$$
\langle a+b, a+b\rangle+\langle a-b, a-b\rangle=2(\langle a, a\rangle+\langle b, b\rangle) .
$$

Therefore,

$$
2\|\langle a, a\rangle+\langle b, b\rangle\| \leq\|a+b\|^{2}+\|a-b\|^{2} .
$$


This completes the proof.

Theorem 1. If $T \in L(E)$ be such that

$$
\inf _{\|x\|=1}\|T x\|^{2}+\left\|T^{*} x\right\|^{2} \leq\left\|\langle T x, T x\rangle+\left\langle T^{*} x, T^{*} x\right\rangle\right\|
$$

and

$$
\inf _{\|x\|=1}\left\|T^{*} x\right\|^{2}+\|T x\|^{2} \leq\left\|\langle T x, T x\rangle+\left\langle T^{*} x, T^{*} x\right\rangle\right\|
$$

for all $x \in E$ with $\|x\|=1$; then

$$
\|T\|^{2}+M(T)-m(T) \leq 2 \delta^{2}(T) .
$$

Proof. Suppose that $u \in E$ with $\|u\|=1$. Choose $a=T u, b=T^{*} u$ in (13) to give

$$
\left\|\langle T u, T u\rangle+\left\langle T^{*} u, T^{*} u\right\rangle\right\| \leq \frac{1}{2}\left(\left\|T u+T^{*} u\right\|^{2}+\left\|T u-T^{*} u\right\|^{2}\right) .
$$

By the assumption, $\inf _{\|x\|=1}\|T x\|^{2}+\left\|T^{*} u\right\|^{2} \leq\left\|\langle T u, T u\rangle+\left\langle T^{*} u, T^{*} u\right\rangle\right\|$ gives

$$
\inf _{\|x\|=1}\|T x\|^{2}+\left\|T^{*} u\right\|^{2} \leq \frac{1}{2}\left(\left\|T u-T^{*} u\right\|^{2}+\left\|T u+T^{*} u\right\|^{2}\right) .
$$

Taking the supremum over $u \in E$ with $\|u\|=1$ gives

$$
\inf _{\|x\|=1}\|T x\|^{2}+\|T\|^{2} \leq \frac{1}{2}\left(\left\|T-T^{*}\right\|^{2}+\left\|T+T^{*}\right\|^{2}\right) . \quad \quad\left(\text { since }\|T\|=\left\|T^{*}\right\|\right)
$$

Since $\left(T+T^{*}\right)$ is self-adjoint, (6) yields

$$
\left\|T+T^{*}\right\| \leq 2 \delta(T)
$$

Therefore,

$$
\inf _{\|x\|=1}\|T x\|^{2}+\|T\|^{2} \leq 2 \delta^{2}(T)+\frac{\left\|T-T^{*}\right\|^{2}}{2} .
$$

Similarly, by the assumption,

$$
\inf _{\|x\|=1}\left\|T^{*} x\right\|^{2}+\|T x\|^{2} \leq\left\|\langle T x, T x\rangle+\left\langle T^{*} x, T^{*} x\right\rangle\right\|,
$$

gives

$$
\inf _{\|x\|=1}\left\|T^{*} x\right\|^{2}+\|T\|^{2} \leq 2 \delta^{2}(T)+\frac{\left\|T-T^{*}\right\|^{2}}{2}
$$


and, so,

$$
\|T\|^{2}+M(T)-\frac{\left\|T-T^{*}\right\|^{2}}{2} \leq 2 \delta^{2}(T) .
$$

Replacing $T$ by $i T$ in the last inequality gives

$$
\|T\|^{2}+M(T)-\frac{\left\|T+T^{*}\right\|^{2}}{2} \leq 2 \delta^{2}(T) .
$$

Thus,

$$
\|T\|^{2}+M(T)-\min \left(\frac{\left\|T-T^{*}\right\|^{2}}{2}, \frac{\left\|T+T^{*}\right\|^{2}}{2}\right) \leq 2 \delta^{2}(T),
$$

which is exactly the desired result.

The following particular case is of interest.

Corollary 1. Let $T$ be as in Theorem 1. If, in addition, $T \in L^{-1}(E)$, then

$$
\|T\|^{2}+\frac{1}{\left\|T^{-1}\right\|^{2}}-m(T) \leq 2 \delta^{2}(T) .
$$

Proof. Result follows immediately from Theorem 1 and Lemma 2(b), since $T$ is invertible.

Our next corollary includes a refinement of the inequality (5).

Corollary. Let $R, S$ be as in Theorem 1. Then

$$
\begin{aligned}
\delta(R S) \leq \sqrt{\left(2 \delta^{2}(R)-M(R)+m(R)\right)\left(2 \delta^{2}(S)-M(S)+m(S)\right)} & \leq \\
& \leq 4 \delta(R) \delta(S)
\end{aligned}
$$

Proof. By Lemma 2(a),

$$
m(R) \leq 2 \delta^{2}(R)
$$

and so

$$
\|R\| \leq \sqrt{2 \delta^{2}(R)-M(R)+m(R)} \leq 2 \delta(R) .
$$

Similarly,

$$
\|S\| \leq \sqrt{2 \delta^{2}(S)-M(S)+m(S)} \leq 2 \delta(S)
$$


Therefore,

$$
\begin{aligned}
& \delta(R S) \leq\|R\|\|S\| \leq \\
& \leq \sqrt{\left(2 \delta^{2}(R)-M(R)+m(R)\right)\left(2 \delta^{2}(S)-M(S)+m(S)\right)} \leq \\
& \leq 4 \delta(R) \delta(S) .
\end{aligned}
$$

The following applications of Theorem 1 improve inequality (4) for some invertible operators.

Corollary. Let $R, S \in L^{-1}(E)$ and satisfy the condition of Theorem 1 . If $m(R) \leq\left\|R^{-1}\right\|^{-2}$ and $m(S) \leq\left\|S^{-1}\right\|^{-2}$, then

$$
\begin{gathered}
\|R\| \leq \sqrt{2} \delta(R), \\
\delta(R S) \leq 2 \delta(R) \delta(S) .
\end{gathered}
$$

Proof. Inequality (18) follows from Lemma 2(b) and corollary 1. Similarly,

$$
\|S\| \leq \sqrt{2} \delta(S)
$$

For inequality (19), observe, using $\delta(R S) \leq\|R S\|$ in the first inequality and (18) and (20) in the third, that

$$
\delta(R S) \leq\|R S\| \leq\|R\|\|S\| \leq 2 \delta(R) \delta(S) .
$$

This completes the proof.

3. New inequalities for Hilbert operators. Since a Hilbert space is a Hilbert $\mathbb{C}^{*}$-module, the results in section 2 of this paper hold in $B(H)$.

Theorem 2. If $T \in B(H)$ and $0<\|T\|^{2}+M(T)-m(T)$, then

$$
\|T\| \leq \sqrt{\frac{2}{1+M\left(\frac{T}{\|T\|}\right)-m\left(\frac{T}{\|T\|}\right)}} \omega(T) .
$$

Proof. According to Definition 1, we have $\delta(T)=\omega(T)$.

Replacing $T$ by $\frac{T}{\|T\|}$ in (14) gives

$$
\|T\|^{2}\left(1+M\left(\frac{T}{\|T\|}\right)-m\left(\frac{T}{\|T\|}\right)\right) \leq 2 \omega^{2}(T) .
$$


Since $\|T\|^{2}+M(T)-m(T)>0$

$$
\|T\|^{2} \leq \frac{2}{1+M\left(\frac{T}{\|T\|}\right)-m\left(\frac{T}{\|T\|}\right)} \omega^{2}(T),
$$

which is exactly the desired result.

In the next result, we provide some conditions for the inequality $\|T\| \leq \sqrt{2} \omega(T)$ to be true.

Corollary. If $T \in B(H)$ and $M(T) \geq m(T)$, then

$$
\|T\| \leq \sqrt{2} \omega(T) .
$$

Acknowledgment. The authors thank the Editorial Board and the referees for their valuable comments that helped to improve the text.

\section{References}

[1] B. E. Cain, Improved inequalities for the numerical radius: when inverse commutes with the norm, Bull. Aust. Math. Soc., 2018, vol. 2, no. 1, pp. 293-296. DOI: https://doi.org/10.1017/S0004972717001046

[2] S. S. Dragomir, Rivers inequalities for the numerical radius of pace, Bull. Aust. Math. Soc., 2006, vol. 73, pp. 255-262.

DOI: https://doi.org/10.1017/S0004972700038831

[3] S. S. Dragomir, Some inequalities of the Grüss type for the Numerical radius of bounded linear operators in Hilbert spaces, J. Inequal. Appl. 2008, Art. ID 763102, 9 p. DOI: https://doi.org/10.1155/2008/763102

[4] S. S. Dragomir, Inequalities for the norm and the numerical radius of linear operators in Hilbert spaces, Demonstratio Mathematica., 2007, vol. 40, no. 2, pp. 411-417. DOI: https://doi.org/10.1515/dema-2007-0213

[5] S. S. Dragomir, Some inequalities for the norm and the numerical radius of linear operators in Hilbert Spaces, Tamkang J. Math., 2008, vol. 39, no. 1, pp. 1-7. DOI: https://doi.org/10.5556/j.tkjm.39.2008.40

[6] K. E. Gustafson and D. K. M. Rao, Numerical Range, Springer-Verlag, New York, 1997.

[7] J. A. R. Holbrook, Multiplicative properties of the numerical radius in operator theory, J. Reine Angew. Math., 1969, vol. 237, pp. 166-174.

DOI: https://doi.org/10.1515/crll.1969.237.166

[8] R. V. Kadison and J. R. Ringrose, Fundamentals of the theory of operator algebras, Vol. 1, Graduate Studies in Mathematics, Amer. Math. Soc. Providence, RI, 1997. 
[9] F. Kittaneh, A numerical radius inequality and an estimate for the numerical radius of the Frobenius companion matrix, Studia Mathematica., 2003, vol. 158, no. 1, pp. 11-17. DOI: https://doi.org/10.4064/sm158-1-2

[10] F. Kittaneh, Numerical radius inequalities for Hilbert space operators, Studia Mathematica., 2005, vol. 168, no. 1, pp. 73-80.

DOI: https://doi.org/10.1155/2009/492154

[11] F. Kittaneh, Numerical radius inequalities for certain $2 \times 2$ operator matrices, Integr. Equ. Oper. Theory., 2011, vol. 71, pp. 129-147.

DOI: https://doi.org/10.1007/s00020-011-1893-0

[12] E. C. Lance, Hilbert $C^{*}$-modules, London Mathematical Society Lecture Note Series, 210, Cambridge University Press, Cambridge, 1995.

[13] M. Shah Hosseini and M. E. Omidvar Some inequalities for the numerical radius for Hilbert space operators, Bull. Aust. Math. Soc., 2016, vol. 94, no. 3, pp. 489-496. DOI: https://doi.org/10.1017/S0004972716000514

[14] M. Shah Hosseini and M. E. Omidvar, Some Reverse and Numerical Radius Inequalities, Math. Slovaca., 2018, vol. 68, no. 5, pp. 1121-1128.

DOI: https://doi.org/10.1515/ms-2017-0174

[15] B. Moosavi and M. Shah Hosseini, Some inequalities for the numerical radius for operators in Hilbert $C^{*}$-modules space, J. Inequ. Special. Func., 2019, vol. 10, no. 1, pp. 77-84.

DOI: https://doi.org/10.1515/gmj-2019-2053

Received December 01, 2019.

In revised form, June 04, 2020.

Accepted June 05, 2020.

Published online June 15, 2020.

M. Shah Hosseini

Department of Mathematics, Shahr-e-Qods Branch, Islamic Azad University, Tehran, Iran.

E-mail: mohsen_shahhosseini@yahoo.com

B. Moosavi

Department of Mathematics, Safadasht Branch, Islamic Azad University,

Tehran, Iran.

E-mail: baharak_moosavie@yahoo.com 